\title{
Magnetosubbands of semiconductor quantum wires with Rashba spin-orbit coupling
}

\author{
J. Knobbe and Th. Schäpers* \\ Institut für Schichten und Grenzfächen and Center of Nanoelectronic Systems for Information Technology, \\ Forschungszentrum Jülich, 52425 Jülich, Germany
}

(Received 6 May 2004; revised manuscript received 2 September 2004; published 12 January 2005)

\begin{abstract}
We have investigated the electronic structure of Rashba spin-split quantum wires in a magnetic field. For our numerical calculations, a harmonic confinement was assumed. We find that wire structures with several occupied one-dimensional subbands still exhibit a beating pattern in the magnetoresistance. The wire width turns out to strongly affect the magnetic field values at which nodes occur in the beating pattern. In the limit of narrow wires, the beating pattern would vanish altogether because spin-split subbands become populated equally.
\end{abstract}

DOI: 10.1103/PhysRevB.71.035311

PACS number(s): 71.70.Ej, 73.63.Nm, 73.61.Ey, 73.43.Qt

\section{INTRODUCTION}

Spin-dependent transport phenomena in semiconductor structures have been studied extensively in recent years because of their potential for future electronic devices. ${ }^{1-4}$ In these devices the spin degree of freedom is used for information processing in addition to the electron charge. A number of devices have been proposed that promise higher speed, lower power consumption, and a higher degree of functionality. ${ }^{5-9}$ Many of these structures rely on the Rashba effect, ${ }^{10,11}$ which allows one to control the spin orientation by means of a gate electrode.

The Rashba spin-orbit coupling originates from a macroscopic electric field in a semiconductor quantum well. The contribution of the Rashba effect to the single-electron Hamiltonian of a two-dimensional electron gas (2DEG) can be expressed as

$$
H_{s o}=\frac{\alpha_{R}}{\hbar} \vec{e}_{z}[\vec{\sigma} \times(\vec{p}-e \vec{A})] .
$$

We have denoted the electron momentum by $\vec{p}$ and the Pauli spin matrices by $\vec{\sigma}$. The magnitude of the Rashba spin-orbit coupling is expressed by the coupling parameter $\alpha_{R}$. The macroscopic electric field originating from the inversion asymmetry in the quantum well is oriented parallel to the growth direction, which is taken to be the $z$ direction. Since we will consider the more general case by including an external magnetic field $\vec{B}$, the kinetic momentum $\vec{p}-e \vec{A}$ was inserted into the Hamiltonian with the vector potential $\vec{A}$ defined by $\vec{B}=\vec{\nabla} \times \vec{A}$.

The Rashba effect is found to be most pronounced in two-dimensional electron gases comprising a low band-gap channel layer (e.g., InAs or InGaAs). The value of the Rashba coupling parameter $\alpha_{R}$ can be extracted from the characteristic beating pattern observed in the magnetoresistance of the two-dimensional electron gas. ${ }^{12-15}$ It could be demonstrated that by applying a voltage to a gate that covers the 2DEG, the effective electric field in the conducting channel and thus the Rashba coupling parameter can be controlled. ${ }^{16-20}$ This property is an important prerequisite for the implementation of the spin transistor proposed by Datta and Das. ${ }^{5}$ However, soon it was realized that for an improved performance of the spin transistor, a restriction to a onedimensional channel is desirable. In addition, many concepts of spin electronic devices rely on a carrier transport restricted to only one dimension. ${ }^{21-24}$

The effect of the Rashba spin-orbit coupling on the transport in one-dimensional systems at zero magnetic field was theoretically investigated by Moroz and Barnes ${ }^{25}$ and Mireles and Kirczenow. ${ }^{26}$ Experimentally it could be demonstrated that similar to the case of a 2DEG the presence of the Rashba effect leads to a characteristic beating pattern in the magnetoresistance. ${ }^{27-30}$ However, for narrow wires it was observed that the nodes in the beating pattern are shifted with respect to the nodes observed in the Shubnikov-de Haas oscillations of the corresponding two-dimensional reference sample. Two mechanisms have been made out as possible explanations for the shift of the nodes: first, an enhanced Rashba spin-orbit coupling, due to a modified effective electric field in the quantum well, and, second, the effect the carrier confinement potential has on the spectrum of the magneto-subbands. ${ }^{29,30}$

In this paper we will demonstrate that the quantization due to the carrier confinement in a one-dimensional system with Rashba spin-orbit coupling results in a modification of the beating pattern of the magnetorestistance, if compared to the case of a 2DEG. The beating pattern is deduced from numerical calculation of the subband spectrum of the wire as function of the electron wave vector of an external magnetic field. The lateral confinement of the quantum wire is modeled by a parabolic potential. We will argue that for a wire structure the number of nodes in the beating is determined by the strength of the confinement potential. At moderate confinement potentials with several tens of sublevels occupied, only a few nodes are expected. If the carrier confinement is sufficiently strong so that only a few subbands are occupied, the beating pattern in the magnetorestistance is even expected to vanish completely. This is in strong contrast to the case of a high-mobility two-dimensional electron gas where a large number of nodes is observed, with the appearance of the highest-order node limited by the characteristic magnetic field determined from the quantum scattering time. ${ }^{13,18,31}$

Below, we will first introduce our model describing the Rashba spin-orbit coupling in a quantum wire. In Sec. III 


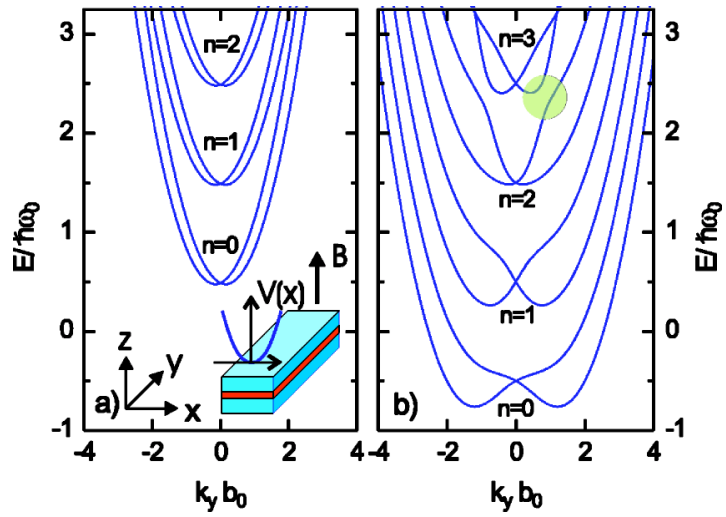

FIG. 1. (Color online) Energy dispersion of the spin-split subbands at $B=0$ for weak spin-orbit coupling: $\Delta_{s o} / \hbar \omega_{0}=0.01$ (a) and for strong spin-orbit coupling for $\Delta_{s o} / \hbar \omega_{0}=1$ (b). The anticrossing between the second and third subband is marked by a circle. The quantity $b_{0}=\sqrt{\hbar / m * \omega_{0}}$ is the characteristic length scale of the harmonic confinement potential. The inset shows the geometry of the wire structure.

some general properties of the sublevel spectrum will be discussed, followed by simulations of the expected node positions in the magnetoresistance under different conditions (i.e., confinement potential, Fermi energy, and Rashba coupling parameter). Conclusions are presented in Sec. IV.

\section{MODEL}

We considered a system where the electrons are confined in a quantum well, with a confining potential determined by the layer sequence of a semiconductor heterostructure (see Fig. 1, inset). The growth direction is taken to be along the $z$ direction. For simplicity only the lowest subband of the quantum well is assumed to be occupied. Regarding the description of the electron propagation we restrict ourself to the $x y$ plane. The magnetic field shall be oriented along the growth direction $\vec{B}=(0,0, B)$. Thus, in the Landau gauge the corresponding vector potential can be expressed by $\vec{A}$ $=B x \vec{e}_{y}$. The single-particle Hamiltonian is then given by $H_{2 D}=H_{0}+H_{s o}$ with

$$
\begin{gathered}
H_{0}=\frac{1}{2 m^{*}}\left[p_{x}^{2}+\left(p_{y}-e B x\right)^{2}\right] \sigma_{0}+V(x) \sigma_{0}+\frac{1}{2} g \mu_{B} \sigma_{z} B, \\
H_{s o}=\frac{\alpha_{R}}{\hbar}\left[\sigma_{x}\left(p_{y}-e B x\right)-\sigma_{y} p_{x}\right],
\end{gathered}
$$

where $\sigma_{0}$ stands for the unit matrix. In $H_{0}$ the first term is the kinetic contribution with $m^{*}$ the effective electron mass. The confining potential of the quantum wire is represented by $V(x)$, while the last term in $H_{0}$ is the Zeeman energy splitting with $\mu_{B}$ the Bohr magneton and $g$ the gyromagnetic factor. Regarding $H_{s o}$, the effect of an external magnetic field was included by inserting the vector potential $\vec{A}$, as defined above. For the confinement of the quantum wire we choose a parabolic potential expressed by

$$
V(x)=\frac{1}{2} m^{*} \omega_{0}^{2} x^{2},
$$

with the oscillator frequency given by $\omega_{0}$. For relatively narrow quantum wires with a surface depletion layer at the edge, this should be a good approximation. ${ }^{32}$ In principle, the lateral confining potential results in a second contribution to the spin-orbit coupling in addition to the contribution arising from the confining potential of the semiconductor heterostructure. However, for the situation considered here with relatively wide wire structures and a weak parabolic confinement potential, the resulting electric field is about three orders-of-magnitude smaller than typical electric fields in asymmetric quantum wells. ${ }^{18}$ This is in agreement to the estimates of Moroz and Barnes, ${ }^{25}$ who also found that for typical electric fields originating from the parabolic confinement potentials, the corresponding spin-orbit coupling parameter is considerably smaller than $\alpha_{R}$. Thus for the situation addressed here, the spin-orbit coupling resulting from the confining potential can be neglected.

With the ansatz

$$
\Psi(x, y)=\phi(x) \exp \left(i k_{y} y\right),
$$

the Schrödinger equation becomes separable in $x$ and $y$. Plane waves are taken along the wire, with wave numbers $k_{y}$ quantized in units of $2 \pi / L$. The quantity $L$ is the wire length. By applying the ansatz, as given by Eq. (5), the Hamiltonian $H_{0}$ reduces to $^{33-35}$

$$
\begin{aligned}
H_{0}= & {\left[-\frac{\hbar^{2}}{2 m^{*}} \frac{d^{2}}{d x^{2}}+\frac{1}{2} m * \omega^{2}\left(x-x_{0}\right)^{2}+\frac{\omega_{0}^{2}}{\omega^{2}} \frac{\hbar^{2} k_{y}^{2}}{2 m^{*}}\right] \sigma_{0} } \\
& +\frac{1}{2} g \mu_{B} \sigma_{z} B
\end{aligned}
$$

with $x_{0}=\left(\omega_{c} / \omega\right)^{2}\left(\hbar k_{y} / e B\right)$ the guiding-center coordinate for the harmonic oscillator, $\omega_{c}=e B / m^{*}$ the cyclotron frequency, and $\omega=\left(\omega_{c}^{2}+\omega_{0}^{2}\right)^{1 / 2}$ the effective oscillator frequency. The quantity $m^{*}\left(\omega / \omega_{0}\right)^{2}$ can be interpreted as an effective magnetic mass of the one-dimensional system. ${ }^{35}$ The set of eigenfunctions of $H_{0}$ obtained from the Schrödinger equation for the system without spin-orbit coupling $H_{0} \phi_{n \sigma}(x)$ $=E_{n \sigma}^{(0)} \phi_{n \sigma}(x)$ is given by

$$
\begin{gathered}
\phi_{n \sigma}(x)=\frac{1}{\sqrt{b}} \frac{\pi^{-1 / 4}}{\sqrt{2^{n} n !}} H_{n}\left(\frac{x-x_{0}}{b}\right) \exp \left(-\frac{\left(x-x_{0}\right)^{2}}{2 b^{2}}\right) \chi_{\sigma}, \\
n=0,1,2, \ldots, \quad \sigma= \pm
\end{gathered}
$$

with $b=\sqrt{\hbar / m * \omega}$ the characteristic length of the harmonic oscillator. $H_{n}(x)$ are the Hermite polynomials of integer order $n$, while $\chi_{+}=\left(\begin{array}{l}1 \\ 0\end{array}\right)$ and $\chi_{-}=\left(\begin{array}{l}0 \\ 1\end{array}\right)$ are the spinors for up- and down-spin projected in the $z$ direction, respectively. The corresponding energy eigenvalues are given by

$$
E_{n \pm}^{(0)}=\left[\hbar \omega\left(n+\frac{1}{2}\right)+\frac{\hbar^{2}}{2 m^{*}} \frac{\omega_{0}^{2}}{\omega^{2}} k_{y}^{2} \pm \frac{1}{2} g \mu_{B} B\right] .
$$

With our plane wave ansatz for the $y$ direction, the spin-orbit Hamiltonian $H_{s o}$ can be written as 


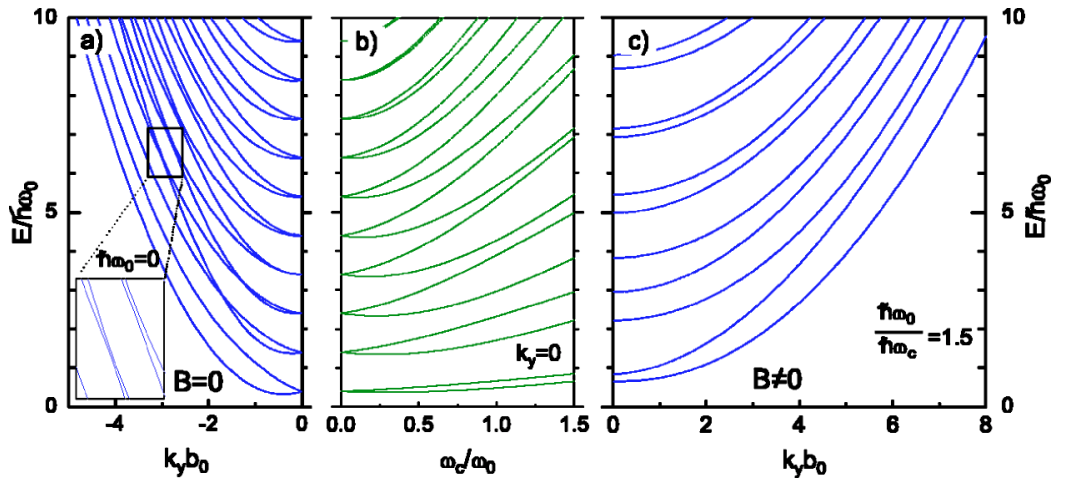

FIG. 2. (Color online) (a) Energy dispersion at zero magnetic field. The inset shows a detail of the anticrossing of the lower subbands. The relative strength of the Rashba spin-orbit coupling $\Delta_{s o} / \hbar \omega_{0}$ was taken to be 0.1 . (b) Energy spectrum at $k_{y}=0$ as a function of $\omega_{c} / \omega_{0}$. For the Zeeman energy splitting $g \mu_{B} B$, we assumed $-0.072 \hbar \omega_{c}$. (c) Energy dispersion at a finite magnetic field $\left(\hbar \omega_{c}=1.5 \hbar \omega_{0}\right)$.

$$
H_{s o}=\alpha_{R}\left[\sigma_{x}\left(k_{y}-\frac{e B}{\hbar} x\right)+i \sigma_{y} \frac{d}{d x}\right] .
$$

By expanding $\phi(x)=\Sigma_{n, \sigma} a_{n \sigma} \phi_{n \sigma}(x)$ in the basis of the eigenfunctions, as given by Eq. (7), the following equations are found:

$$
\left(E_{n \sigma}^{(0)}-E\right) a_{n \sigma}+\sum_{m, \sigma^{\prime} \neq \sigma}\left(H_{s o}\right)_{n m}^{\sigma \sigma^{\prime}} a_{m \sigma^{\prime}}=0,
$$

with the matrix elements $\left(H_{s o}\right)_{n m}^{\sigma \sigma^{\prime}}=\left\langle\phi_{n \sigma}\left|H_{s o}\right| \phi_{m \sigma^{\prime}}\right\rangle$ given by

$$
\begin{aligned}
\left(H_{s o}\right)_{n n}^{\sigma \sigma^{\prime}}=-\alpha_{R}\left(\frac{\omega_{c}^{2}}{\omega^{2}}-1\right) k_{y}, \\
\left(H_{s o}\right)_{01}^{ \pm \mp}=\frac{\alpha_{R}}{b \sqrt{2}}\left(\frac{\omega_{c}}{\omega} \pm 1\right), \\
\left(H_{s o}\right)_{n m}^{ \pm \mp}=\frac{\alpha_{R}}{b}\left(\frac{\omega_{c}}{\omega} \pm 1\right) \sqrt{\frac{n+1}{2}} \delta_{n, m-1} \\
+\frac{\alpha_{R}}{b}\left(\frac{\omega_{c}}{\omega} \mp 1\right) \sqrt{\frac{n}{2}} \delta_{n, m+1}, \quad n \geqslant 1 .
\end{aligned}
$$

The matrix elements $\left(H_{s o}\right)_{n n}^{\sigma \sigma^{\prime}}$ couple opposite spins within a given state $n$, whereas $\left(H_{s o}\right)_{01}^{\sigma \sigma^{\prime}}$ and $\left(H_{s o}\right)_{n m}^{\sigma \sigma^{\prime}}$ couple neighboring oscillator-level states with opposite spins. For a given $k_{y}$ the energy eigenvalues for nonzero spin-orbit coupling $\left(\alpha_{R} \neq 0\right)$ are determined by numerically calculating the roots of the set of equations defined by Eq. (10).

Before the numerical results are presented in the following section, we will give some general remarks about the properties of Eq. (10). We will also point out the differences compared to the two-dimensional case. The matrix elements $\left(H_{s o}\right)_{n n}^{\sigma \sigma^{\prime}}$ solely arise from the one-dimensional confinement potential. For a two-dimensional system $\left(\omega_{0}=0\right)$, where the eigenvalues $E_{n \sigma}^{(0)}$ correspond to the Landau level energies, the matrix elements $\left(H_{s o}\right)_{n n}^{\sigma \sigma^{\prime}}$ are zero. Regarding $\left(H_{s o}\right)_{01}^{\sigma \sigma^{\prime}}$ and $\left(H_{s o}\right)_{n m}^{\sigma \sigma^{\prime}}$ only the terms with the prefactor $\left(\omega_{c} / \omega+1\right)$ lead to a nonzero contribution in a 2DEG. In this case the energy eigenvalue spectrum determined from Eq. (10) reduces to the results calculated by Rashba for a two-dimensional system. ${ }^{10}$ At large magnetic fields, where the Landau energy is much larger than the contribution due to the confinement potential $\left(\hbar \omega_{c} \gg \hbar \omega_{0}\right)$, the levels of the wire effectively merge with the Landau levels of a two-dimensional system. ${ }^{34}$

At zero magnetic field and $k_{y}=0$, the spin degeneracy is preserved. However, as pointed out by Häusler ${ }^{36}$ and by Governale and Zülicke, ${ }^{22}$ in the presence of Rashba spinorbit coupling the eigenenergies of the unperturbed system determined from Eq. (10) are uniformly shifted downward by $\Delta_{s o}$, with $\Delta_{s o}=m * \alpha_{R}^{2} / 2 \hbar^{2}$ the characteristic Rasbha spinorbit energy.

\section{SIMULATIONS}

In order to illustrate to what extent the Rashba effect modifies the energy dispersion in a one-dimensional structure, the energy-momentum relation was calculated for weak $\left(\Delta_{s o} / \hbar \omega_{0}=0.01\right)$ and strong $\left(\Delta_{s o} / \hbar \omega_{0}=1\right)$ spin-orbit coupling. In the first case, spin splitting due to the Rashba spinorbit coupling is observed within each subband with a degeneracy point at $k_{y}=0$ [Fig. 1(a)]. However, owing to the weak spin-orbit contribution the coupling between different subbands can be neglected for the energy range considered here. As shown in Fig. 1(b), the situation changes completely if a stronger spin-orbit coupling is assumed. Now, a significant coupling between neighboring subbands is found, due to the larger off-diagonal elements $\left(H_{s o}\right)_{n m}^{ \pm \mp}$. This leads to a pronounced anticrossing, as can be seen in Fig. 1(b) (circle). ${ }^{22,25}$ As outlined above at the degeneracy points at $k_{y}=0$, the Rashba effect results in an energy shift of the subbands by $\Delta_{s o}$ compared to the unperturbed system.

For the following discussion of the beating pattern in the magnetoresistance information is needed about the modification of the sublevel spectrum at finite magnetic fields. In Fig. 2 it is illustrated to what extent the sublevel spectrum is affected by a magnetic field. For the curves shown here, a moderate Rashba spin-orbit coupling strength of $\Delta_{s o} / \hbar \omega_{0}$ $=0.1$ was chosen. As can be seen in Fig. 2(a) at zero magnetic field an anticrossing of the subbands occurs. However, owing to the smaller ratio $\Delta_{s o} / \hbar \omega_{0}$ the anticrossing is much weaker compared to the case shown in Fig 1(b). Applying a magnetic field results in an increased subband separation, which is now given by $\hbar \omega$ according to Eq. (6), with $\omega$ $=\left(\omega_{0}^{2}+\omega_{c}^{2}\right)^{1 / 2}$ instead of $\omega_{0}$ at zero field. The corresponding level spectrum for $k_{y}=0$ as a function of $\omega_{c} / \omega_{0}$ is shown in Fig. 2(b). By applying a magnetic field the time-reversal symmetry is broken. As a consequence the Rashba effect 
results in a lifting of the spin degeneracy at $k_{y}=0$. However, the interplay between the Rashba spin-orbit coupling and the Zeeman splitting leads to a rather complex level spectrum, where at a given magnetic field the energy separation between the different subbands varies. As we will see below, the variation of the level separation is the origin of the characteristic beating pattern, which can be observed in the magnetoresistance. In Fig. 2(c) the normalized energy versus wave-vector dispersion is plotted at a finite magnetic field $\left(\omega_{c}=1.5 \omega_{0}\right)$. In contrast to the zero field case, no anticrossing of the subbands occur in the energy range considered here. We attributed this to the enhanced effective oscillator energy $\hbar \omega$ with reference to $\Delta_{s o}$. Compared to the zero magnetic field the slope of the dispersion is smaller which can be interpreted as an increased effective mass. ${ }^{33,34}$

In order to be able to compare most directly to experimental results, we have to be more specific about the heterostructure. Here, we choose an $\mathrm{In}_{0.53} \mathrm{Ga}_{0.47} \mathrm{As} / \mathrm{In}_{0.77} \mathrm{Ga}_{0.23} \mathrm{As} / \mathrm{InP}$ heterostructure, where a pronounced beating pattern due to the Rashba spin-orbit coupling has been observed in the magnetoresistance of the two-dimensional electron gas. ${ }^{17,18}$ As a typical sheet electron concentration we assumed $n_{2 D}$ $=6 \times 10^{11} \mathrm{~cm}^{-2}$, which corresponds to a Fermi energy of $E_{F, 2 D}=39 \mathrm{meV}$ with $m^{*}=0.037 .{ }^{37}$ For the $g$ factor a value of -4 was assumed. ${ }^{38}$ The value of $E_{F, 2 D}$ was taken as the zero field value of the quantum wire Fermi energy $E_{F, 1 D}$, which should be a good approximation for wire structures with a larger number of occupied levels. In principle, the carrier confinement in the wire can be characterized by the oscillator frequency $\omega_{0}$ if a harmonic confinement potential is assumed. However, since we intend to relate our simulations to the geometrical dimensions of the wire, we rather refer to the effective wire width $w$. By taking $w$ as a given value, the one-dimensional carrier density is determined by $n_{1 D}=w n_{2 D}{ }^{35}$ The oscillator frequency $\omega_{0}$ of the wire is determined iteratively. Here, the subbands corresponding to an assumed value of $\omega_{0}$ are filled with electrons until $n_{1 D}$ is reached. The oscillation frequency $\omega_{0}$ is then adjusted iteratively until the Fermi energy resulting from the filling of the subbands agrees to the target value $E_{F, 1 D}$.

As illustrated in Fig. 2, the energy-momentum relation of the subbands is modified if an external magnetic field is applied. As a consequence, the Fermi energy for given values of $n_{1 D}$ and $\omega$ also varies as a function of a magnetic field. This is shown in Fig. 3, where $w=300 \mathrm{~nm}$ and a Rashba coupling parameter of $\alpha_{R}=8 \times 10^{-12} \mathrm{eV} \mathrm{m}$ were assumed. However, as can be seen here, in the magnetic field range in which we are interested $(B \leqslant 1 \mathrm{~T})$, the Fermi energy only deviates by $\approx 1 \%$ from the zero field value. In order to simplify the calculations, a constant Fermi energy fixed at the zero field value was assumed in the following simulations.

The oscillations in the magnetoresistance of a quantum wire are directly related to the periodic structure in the density of states. ${ }^{35}$ In order to clarify the origin of the characteristic beating pattern in the magnetoresistance in the presence of the Rashba effect, the density of states at the Fermi energy as a function of the magnetic field was determined from the subband dispersion. Figure 4(a) shows the density of states of a $500 \mathrm{~nm}$ wide wire. For the Rashba coupling parameter a value of $8 \times 10^{-12} \mathrm{eVm}$ was assumed. By increasing the

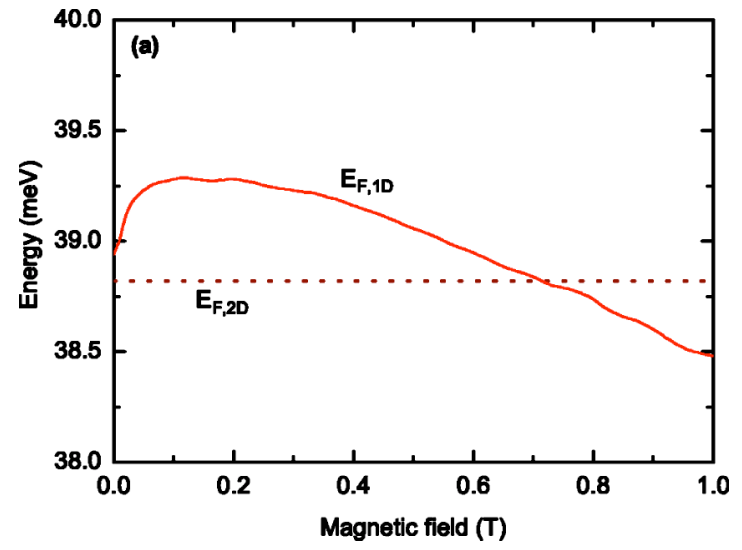

FIG. 3. (Color online) Fermi energy as a function of the magnetic field for a $300 \mathrm{~nm}$ wide wire. For the Rashba coupling parameter a value of $8 \times 10^{-12} \mathrm{eV} \mathrm{m}$ was assumed.

magnetic field, the magnetoelectric subbands are successively depopulated. Each time the bottom of a subband $\left(k_{y}\right.$ $\approx 0$ ) crosses the Fermi energy, a peak is found in the density of states. Note that at particular magnetic fields for a given peak in the density of states, the distance to the neighboring peaks on the left and right sides is almost identical (i.e., at $B=0.17 \mathrm{~T}$ and $0.63 \mathrm{~T}$ ). At these magnetic field values a node in the magnetoresistance oscillations is expected. The appearance of the beating pattern can directly be visualized if a broadening of the energy levels due to scattering is included. The density of states shown in Fig. 4(b) was obtained by assuming a lifetime broadening described by Lorentz distribution function with a scattering parameter $\Gamma=0.4 \mathrm{meV}$. Now a clear beating pattern in the density of states can be observed with nodes appearing at about $0.17 \mathrm{~T}$ and $0.63 \mathrm{~T}$. A closer inspection of the oscillations near $B=0 \mathrm{~T}$ reveals an increased oscillation period. This can be attributed to the additional contribution of the confinement energy compared to the bare Landau quantization. The oscillation amplitude is relatively large because of the overlap of the two spin-split peaks in the density of states. In general, the oscillations in the magnetoresistance will qualitatively follow the oscilla-

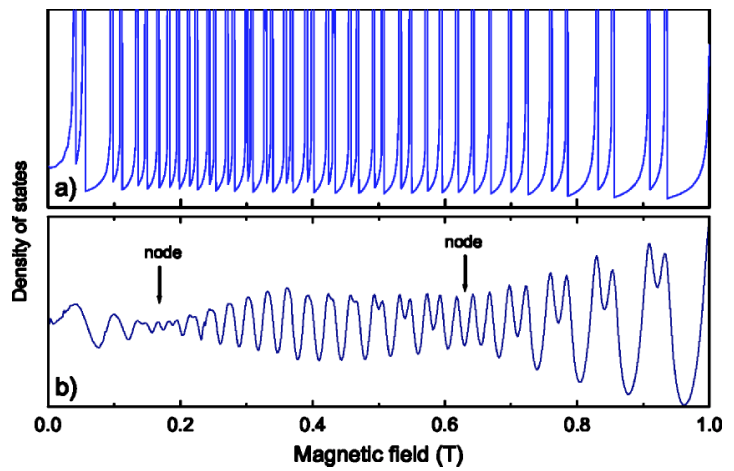

FIG. 4. (Color online) (a) One-dimensional density of states of a $500 \mathrm{~nm}$ wide wire as a function of a magnetic field at a fixed Fermi energy of $39 \mathrm{meV}$. For the Rashba coupling parameter a value of $7.5 \times 10^{-12} \mathrm{eV}$ m was assumed. (b) Density of states for a lifetime broadened energy spectrum. For the scattering parameter $\Gamma$ a value of $0.4 \mathrm{meV}$ was assumed. 


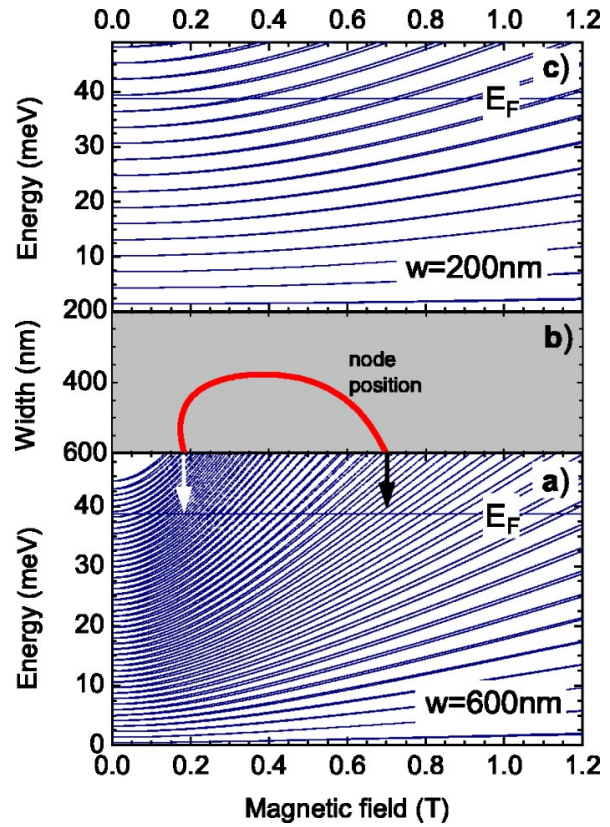

FIG. 5. (Color online) (a) and (c) subband spectra at $k_{y}=0$ as a function of the magnetic field for a 600 and $200 \mathrm{~nm}$ wide wire, respectively. Here, a Rashba coupling parameter of 7.5 $\times 10^{-12} \mathrm{eV} \mathrm{m}$ was assumed. (b) The position of the nodes in the magnetoresistance as a function of the wire width.

tions found in the density of states. However, a detailed calculation of the magnetoresitance of a quantum wire (e.g., as outlined by Tso and Vasilopolous ${ }^{39}$ ) is omitted here for the sake of simplicity.

By using the procedure outlined above we can now address our main question of how the beating pattern in the magnetoresistance is modified if the confinement in the wire is varied. For this purpose the magnetoelectric-subband spectrum has been calculated for different effective wire widths. For example, the subband spectra for two different wire dimensions, namely, $600 \mathrm{~nm}$ and $200 \mathrm{~nm}$, are depicted in Figs. 5(a) and 5(b). The spectra are calculated at $k_{y}=0$ for $\alpha_{R}$ $=7.5 \times 10^{-12} \mathrm{eV} \mathrm{m}$. As explained above, the modulations in the magnetoresistance of the quantum wire are determined by the density of states at the Fermi level. A maximum in the resistance is expected if an enhanced density of states is found at $E_{F, 1 D}$. This is the case if the bottom of a magnetoelectric subband at $k_{y} \approx 0$, crosses the Fermi level. Since the Fermi energy depends only negligibly on the magnetic field, $E_{F, 1 D}$ was assumed to be constant in the following. As mentioned above, a node in the magnetoresistance is expected at a magnetic field where the subbands crossing the Fermi level are evenly spaced. For the $600 \mathrm{~nm}$ wide wire, this situation is found at $0.18 \mathrm{~T}$ and $0.70 \mathrm{~T}$ [indicated by arrows in Fig. $5(\mathrm{a})$. In contrast, if two sublevels approach each other, which is the case in between the node positions [see Fig. $5(\mathrm{a})$, an enhanced modulation amplitude is expected in the magnetoresistance. In contrast to a two-dimensional system, only two nodes are found for the $600 \mathrm{~nm}$ wide wire. This can be explained by the fact that at low magnetic fields the geometrical confinement is the leading contribution to the level spacing. However, even at higher fields the confinement po-

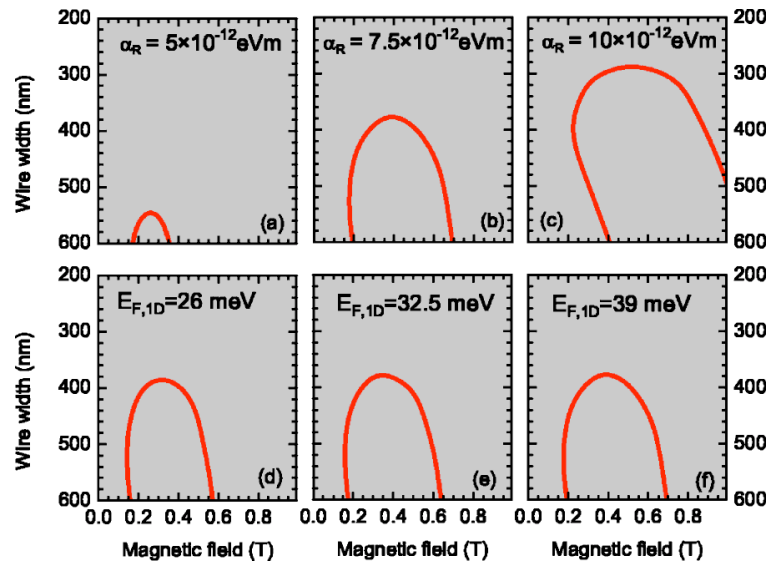

FIG. 6. (Color online) (a)-(c) Expected node positions as a function of wire width with $E_{F, 1 D}=39 \mathrm{meV}$ for $\alpha_{R}=5$ $\times 10^{-12} \mathrm{eV} \mathrm{m}, 7.5 \times 10^{-12} \mathrm{eV} \mathrm{m}$, and $10 \times 10^{-12} \mathrm{eV} \mathrm{m}$, respectively. (d)-(f) Node positions for $E_{F, 1 D}=26 \mathrm{meV}, 32.5 \mathrm{meV}$, and $39 \mathrm{meV}$, respectively. The Rashba coupling parameter was kept at $7.5 \times 10^{-12} \mathrm{eV} \mathrm{m}$.

tential cannot be neglected. For the corresponding twodimensional electron gas, the first two nodes are expected at $0.77 \mathrm{~T}$ and $0.41 \mathrm{~T}$, which deviate significantly from the values given above.

For the $600 \mathrm{~nm}$ wide wire, the corresponding sublevel spacing $\hbar \omega_{0}$ is $0.9 \mathrm{meV}$, whereas for the $200 \mathrm{~nm}$ wide wire, we determined a level separation of $2.9 \mathrm{meV}$. As can be seen in Fig. 5(c), this enhanced confinement energy $\hbar \omega_{0}$ leads to a situation where only a negligible spin splitting of the sublevels occurs. Consequently, no node in the modulation of the magnetoresistance is expected. In Fig. 5(b) the position of the nodes are plotted as a function of the wire width. It can be seen that first the two nodes are shifted toward lower magnetic fields if the wire width is reduced. At a wire width of about $400 \mathrm{~nm}$ both nodes merge and are expected to disappear if the wire width is reduced further.

The node positions strongly depend on the magnitude of the Rashba coupling parameter. This is illustrated in Figs. 6(a)-6(c), where for increasing $\alpha_{R}$ the separation of the two nodes enlarges and shifts toward larger magnetic fields, similar to the case of a two-dimensional electron gas. Although the separation of the nodes increases for larger spin-orbit coupling parameters, the number of oscillations in between is decreasing. This, at first sight, peculiar behavior results from the fact that the oscillations are approximately periodic in $1 / B$ and that for larger values of $\alpha_{R}$ the first node already appears at smaller values of $1 / B$. For larger values of $\alpha_{R}$, the beating pattern in the magnetoresistance should be observable down to smaller wire widths because of the larger characteristic energy $\Delta_{s o}$ relative to the confinement energy $\hbar \omega_{0}$. In contrast, a variation of the Fermi energy by keeping $\alpha_{R}$ at a constant value does not significantly change the threshold of the wire width where a beating pattern appears [as can be seen in Figs. 6(d)-6(f)]. The major effect of an increased Fermi energy is an increase of the node separation.

\section{CONCLUSION}

In conclusion, we have considered the effect of the Rashba spin-orbit coupling on the energy dispersion of a 
quantum wire structure. A harmonic potential was chosen to model the lateral carrier confinement. We focused particular attention on the effect of an external magnetic field on the subband dispersion. In general, an external magnetic field results in an increased effective oscillator frequency and, thus, in an increased separation of the one-dimensional subbands. As a consequence, the coupling between neighboring subbands due to the Rashba effect is weakened. The spectrum at zero field gets strongly rearranged when a magnetic field is switched on. For wider quantum wire structures with many occupied subbands, a beating pattern is expected in the magnetoresistance. However, the number of nodes of the beating pattern is reduced, due to the effect of the geometrical confinement at lower magnetic fields. Compared to a two-dimensional electron gas, the node positions of the wire structures are shifted. Therefore, care has to be taken when extracting the Rashba coupling constant from the node positions because the methods used for two-dimensional structures cannot be applied directly. ${ }^{12-15}$ For very narrow wires, the beating pattern in the magnetoresistance is expected to vanish altogether.

\section{ACKNOWLEDGMENT}

We are indebted to U. Zülicke (Massey University, New Zealand) for fruitful discussions about the Rashba effect in low-dimensional structures.
*Electronic address: th.schaepers@fz-juelich.de

${ }^{1}$ Y. Ohno, Science 281, 951 (1998).

${ }^{2}$ G. A. Prinz, Science 282, 1660 (1998).

${ }^{3}$ P. Ball, Nature (London) 404, 918 (2000).

${ }^{4}$ S. A. Wolf, Science 294, 1488 (2001).

${ }^{5}$ S. Datta and B. Das, Appl. Phys. Lett. 56, 665 (1990).

${ }^{6}$ E. de Andrada e Silva and G. Rocca, Phys. Rev. B 59, R15 583 (1999).

${ }^{7}$ T. Koga, J. Nitta, H. Takayanagi, and S. Datta, Phys. Rev. Lett. 88, 126601 (2002).

${ }^{8}$ J. C. Egues, G. Burkard, and D. Loss, Appl. Phys. Lett. 82, 2658 (2003).

${ }^{9}$ J. Schliemann, J. C. Egues, and D. Loss, Phys. Rev. Lett. 90, 146801 (2003).

${ }^{10}$ E. I. Rashba, Fiz. Tverd. Tela (Leningrad) 2, 1224 (1960) [Sov. Phys. Solid State 2, 1109 (1960)].

${ }^{11}$ Y. Bychkov and E. I. Rashba, J. Phys. C 17, 6039 (1984).

${ }^{12}$ J. Luo, H. Munekata, F. F. Fang, and P. J. Stiles, Phys. Rev. B 38, 10142 (1988).

${ }^{13}$ B. Das, D. C. Miller, S. Datta, R. Reifenberger, W. P. Hong, P. K. Bhattacharya, J. Singh, and M. Jaffe, Phys. Rev. B 39, 1411 (1989).

${ }^{14}$ B. Das, S. Datta, and R. Reifenberger, Phys. Rev. B 41, 8278 (1990).

${ }^{15}$ J. Luo, H. Munekata, F. F. Fang, and P. J. Stiles, Phys. Rev. B 41, 7685 (1990).

${ }^{16}$ J. Nitta, T. Akazaki, H. Takayanagi, and T. Enoki, Phys. Rev. Lett. 78, 1335 (1997).

${ }^{17}$ G. Engels, J. Lange, Th. Schäpers, and H. Lüth, Phys. Rev. B 55, R1958 (1997).

${ }^{18}$ Th. Schäpers, G. Engels, J. Lange, Th. Klocke, M. Hollfelder, and H. Lüth, J. Appl. Phys. 83, 4324 (1998).

${ }^{19}$ Y. Sato, T. Kita, S. Gozu, and S. Yamada, J. Appl. Phys. 89, 8017
(2001).

${ }^{20}$ D. Grundler, Phys. Rev. Lett. 84, 6074 (2000).

${ }^{21}$ A. A. Kiselev and K. W. Kim, Appl. Phys. Lett. 78, 775 (2001).

${ }^{22}$ M. Governale and U. Zülicke, Phys. Rev. B 66, 073311 (2002).

${ }^{23}$ M. Governale, D. Boese, U. Zülicke, and C. Schroll, Phys. Rev. B 65, 140403 (2002).

${ }^{24}$ U. Zülicke and M. Governale, Phys. Rev. B 65, 205304 (2002).

${ }^{25}$ A. V. Moroz and C. H. W. Barnes, Phys. Rev. B 61, R2464 (2000).

${ }^{26}$ F. Mireles and G. Kirczenow, Phys. Rev. B 64, 024426 (2001).

${ }^{27}$ Y. Sato, S. Gozu, T. Kikutani, and S. Yamada, Physica B 272, 114 (1999).

${ }^{28}$ Th. Schäpers, J. Knobbe, A. van der Hart, and H. Hardtdegen, Sci. Technol. Adv. Mater. 4, 1925 (2003).

${ }^{29}$ Th. Schäpers, J. Knobbe, V. A. Guzenko, and H. Hardtdegen, Physica E (Amsterdam) 21, 933936 (2004).

${ }^{30}$ Th. Schäpers, J. Knobbe, and V. A. Guzenko, Phys. Rev. B 69, 235323 (2004).

${ }^{31}$ Y. S. Gui, C. Hu, Z. Chen, G. Zheng, S. L. Guo, J. Chu, J. X. Chen, and A. Z. Li, Phys. Rev. B 61, 7237 (2000).

${ }^{32}$ S. E. Laux, D. J. Frank, and F. Stern, Surf. Sci. 196, 101 (1988).

${ }^{33}$ K. F. Berggren, T. J. Thornton, D. J. Newson, and M. Pepper, Phys. Rev. Lett. 57, 1769 (1986).

${ }^{34}$ S. B. Kaplan and A. Hartstein, Phys. Rev. Lett. 56, 2403 (1986).

${ }^{35}$ K.-F. Berggren, G. Roos, and H. van Houten, Phys. Rev. B 37, 10118 (1988).

${ }^{36}$ W. Häusler, Phys. Rev. B 63, 121310(R) (2001).

${ }^{37}$ Th. Schäpers, J. Appenzeller, H. Hardtdegen, and H. Lüth, J. Appl. Phys. 83, 2360 (1998).

${ }^{38}$ M. Dobers, J. P. Vieren, Y. Guldner, P. Bove, F. Omnes, and M. Razeghi, Phys. Rev. B 40, 8075 (1989).

${ }^{39}$ H. C. Tso and P. Vasilopolous, Phys. Rev. B 44, 12952 (1991). 